\title{
Functional, structural, and metabolic abnormalities of the hippocampal formation in Williams syndrome
}

\author{
Andreas Meyer-Lindenberg,1,2,3 Carolyn B. Mervis,, Deepak Sarpal,1,3 Paul Koch,1,3 Sonya Steele,2,3 \\ Philip Kohn, 1,3 Stefano Marenco, ${ }^{2,3}$ Colleen A. Morris, ${ }^{5}$ Saumitra Das, ${ }^{2,3}$ Shane Kippenhan, ${ }^{1,3}$ \\ Venkata S. Mattay, ${ }^{2,3}$ Daniel R. Weinberger, ${ }^{3}$ and Karen Faith Berman ${ }^{1,3}$

\begin{abstract}
${ }^{1}$ Section on Integrative Neuroimaging, ${ }^{2}$ Neuroimaging Core Facility, and ${ }^{3}$ Clinical Brain Disorders Branch, Genes, Cognition, and Psychosis Program, National Institute of Mental Health, NIH, Department of Health and Human Services, Bethesda, Maryland, USA.

${ }^{4}$ Neurodevelopmental Sciences Laboratory, Department of Psychological and Brain Sciences, University of Louisville, Louisville, Kentucky, USA. 5Department of Pediatrics, University of Nevada School of Medicine, University of Nevada, Las Vegas, Nevada, USA.
\end{abstract}

\begin{abstract}
Williams syndrome (WS), caused by microdeletion of some 21 genes on chromosome $7 \mathrm{q} 11.23$, is characterized by dysmorphic features, mental retardation or learning difficulties, elastin arteriopathy, and striking neurocognitive and social-behavioral abnormalities. Recent studies of murine knockouts of key genes in the microdeleted region, LIM kinase 1 (LIMK1) and cytoplasmatic linker protein 2 (CYLN2), demonstrated significant functional and metabolic abnormalities, but grossly normal structure, in the hippocampal formation (HF). Furthermore, deficits in spatial navigation and long-term memory, major cognitive domains dependent on hippocampal function, have been described in WS. We used multimodal neuroimaging to characterize hippocampal structure, function, and metabolic integrity in 12 participants with WS and 12 age-, sex-, and IQ-matched healthy controls. PET and functional MRI studies showed profound reduction in resting blood flow and absent differential response to visual stimuli in the anterior HF in WS. Spectroscopic measures of $\mathrm{N}$-acetyl aspartate, considered a marker of synaptic activity, were reduced. Hippocampal size was preserved, but subtle alterations in shape were present. These data demonstrate abnormalities in HF in WS in agreement with murine models, implicate LIMK1 and CYLN2 in human hippocampal function, and suggest that hippocampal dysfunction may contribute to neurocognitive abnormalities in WS.
\end{abstract}

\section{Introduction}

Williams (or Williams-Beuren) syndrome (WS) (Online Mendelian inheritance in man [OMIM]; 194050) is a neurodevelopmental microdeletion disorder with an estimated prevalence of 1 in 7,500 (1). Besides characteristic cardiovascular abnormalities (particularly supravalvular aortic stenosis), transient juvenile hypercalcemia, and typical facial features, individuals with WS show a unique neurological and behavioral profile that encompasses mild to moderate mental retardation and impaired visuospatial constructive cognition accompanied by relative strengths in verbal short-term memory and language (2), hypersociability, and increased nonsocial anxiety (3). Neurologically, individuals with WS display coordination problems (e.g., difficulty walking up or down a staircase), hyperreflexia, hypersensitivity to sound, and nystagmus (4). WS results from a hemizygous deletion, caused by unequal homologous recombination at flanking repeats during meiosis, of approximately $1.6 \mathrm{Mb}$ on chromosome band 7q11.23 (5). This region contains at least 21 genes, including those encoding elastin (ELN), LIM kinase 1 (LIMK1), and a cytoplasmic linker protein of $115 \mathrm{kDa}$ (CLIP-115)

Nonstandard abbreviations used: BOLD, blood oxygen level-dependent contrast; Cho, choline; CLIP-115, cytoplasmic linker protein of $115 \mathrm{kDa}$; Cre, creatine; CYLN2, cytoplasmatic linker protein 2; F[1,25], F-test with 1,25 degrees of freedom; fMRI, functional MRI; HF, hippocampal formation; LIMK1, LIM kinase 1; MRS, magnetic resonance spectroscopy; MRSI, MRS imaging; NAA, $N$-acetyl aspartate; rCBF, regional cerebral blood flow; ROI, region of interest; WS, Williams syndrome.

Conflict of interest: The authors have declared that no conflict of interest exists.

Citation for this article: J. Clin. Invest. 115:1888-1895 (2005).

doi:10.1172/JCI24892. (cytoplasmic linker protein 2 [CYLN2]). Study of individuals with smaller than typical deletions or point mutations has established that haploinsufficiency for elastin is linked to cardiovascular abnormalities in WS (6). Candidate genes for neurobehavioral abnormalities include LIMK1, implicated by linkage in the genesis of the visuospatial constructive deficit (7), and CYLN2 (8). Both genes code proteins that regulate dynamic aspects of the cytoskeleton of the cell (9). The LIMK1 gene encodes a cytoplasmic protein kinase that is prominently expressed in the developing brain (10). LIMK1 controls actin dynamics via phosphorylation of cofilin and has recently been implicated in the control of growth cone motility in cultured neurons (9). The CYLN2 gene encodes a cytoplasmic linker protein of $115 \mathrm{kDa}$ (CLIP-115), implicated in the local regulation of microtubule dynamics, especially in response to positional cues (8). These alterations may lead to defects in neuronal structure and synaptic plasticity in adulthood and/or to defects during brain development. Knockout mouse models for both of these genes have recently been described $(8,11)$. In each case, phenotypes were characterized by prominent functional alterations of hippocampal formation (HF) in the setting of grossly normal structure. In particular, both knockout mouse models showed changes in hippocampus-dependent learning and hippocampal physiology: in the heterozygous CYLN2 knockout, hippocampal long-term potentiation was reduced (8) while in the homozygous LIMK1 knockout, long-term potentiation was reduced at low- and increased at high-stimulation frequencies (11). In addition, the morphology of dendritic spines of pyramidal neurons in the HF was abnormal in LIMK1 knockouts: spines had thicker necks but smaller head regions (11). 
Table 1

Hippocampal size

\begin{tabular}{lcc} 
& Left hippocampus $\left(\mathbf{m m}^{3}\right)$ & Right hippocampus $\left(\mathbf{m m}^{\mathbf{3}}\right)$ \\
WS & $3259.9 \pm 110.8$ & $3349.3 \pm 137.1$ \\
Controls & $3300.4 \pm 114.9$ & $3452.0 \pm 142.3$ \\
\hline
\end{tabular}

These preclinical results strongly suggest involvement of the HF in the pathophysiology of WS. The functional status of this region in WS is also of great interest in cognitive neuroscience. Decades of work in rodents (12), nonhuman primates, and human primates (13) have shown that the hippocampal region (cornu ammonis fields, dentate gyrus, and subicular complex) and the adjacent cortices are crucial both for declarative memory (conscious memory of facts and events) and for processing of spatial navigational information (14). For both functions, the hippocampus is critically dependent on convergent projections from and to perirhinal and parahippocampal cortices, which in turn are extensively bidirectionally connected with much of the neocortex (15). In WS, there is clinical evidence for impairment of both functional domains: 2 studies found significant impairment of verbal long-term memory and mental retardation in participants with WS compared with normally developing controls $(16,17)$, and spatial cognition and navigational abilities were shown to be markedly impaired $(18,19)$.

Despite these strong convergent preclinical and clinical studies, no human data on the physiology and functional activity of the hippocampus in WS are available. This is partly due to the intellectual impairment associated with the syndrome, precluding comparison with a control group of matched intelligence and reducing the ability of participants with WS to perform consistently during testing. In the present study, we avoided this problem by examining a highly select group of normalintelligence participants with WS (20), expecting that abnormalities found even in this high-performing group would be close to the genetic substrate of the disorder. We adopted a multimodal imaging approach for comprehensively characterizing the hippocampal region. We mapped baseline neurofunctional activity using resting-state positron $\mathrm{O}^{15}-\mathrm{H}_{2} \mathrm{O}$ PET to measure regional cerebral blood flow (rCBF). Synaptic abundance and activity of the hippocampal region were assessed indirectly using proton magnetic resonance spectroscopy (MRS) to assay in vivo the cellular integrity marker $N$-acetyl aspartate (NAA), which is produced primarily in neu- rons and is related to mitochondrial oxidative phosphorylation (21). The functional response of the HF was studied with functional MRI (fMRI), using face and house visual stimuli, which differ in their relevance with regard to spatial cognition and to which differential response of the hippocampus in controls has been shown (22). Finally, we investigated the structure of the HF manually delineated on high-resolution structural MRIs, measuring both overall volume and changes in shape using deformation-based morphometry (23). The latter method shows local changes in shape and size by spatially normalizing individual hippocampi to a same-target template and then analyzing the parameters describing the deformations (the shape changes) that ensue. We hypothesized that our results would demonstrate pronounced functional abnormality, but grossly preserved structure, of the HF in WS.

\section{Results}

Structural brain imaging. Structural neuroimaging was used to directly measure hippocampal volumes and assess changes in shape between participant groups, using deformation-based morphometry. Measured hippocampal volumes are listed in Table 1. The volumes of the manually delineated hippocampi did not differ between groups [main effect of diagnostic group: F-test with 1,25 degrees of freedom $(F[1,25])=0.18, P=0.68]$. While right hippocampi were significantly larger across both groups $(\mathrm{F}[1,25]=5.0$, $P<0.04)$, there was no statistical interaction between laterality and

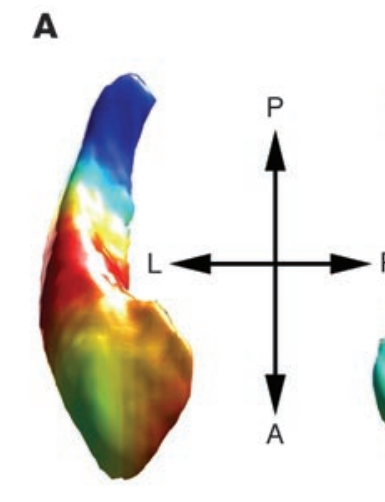

C
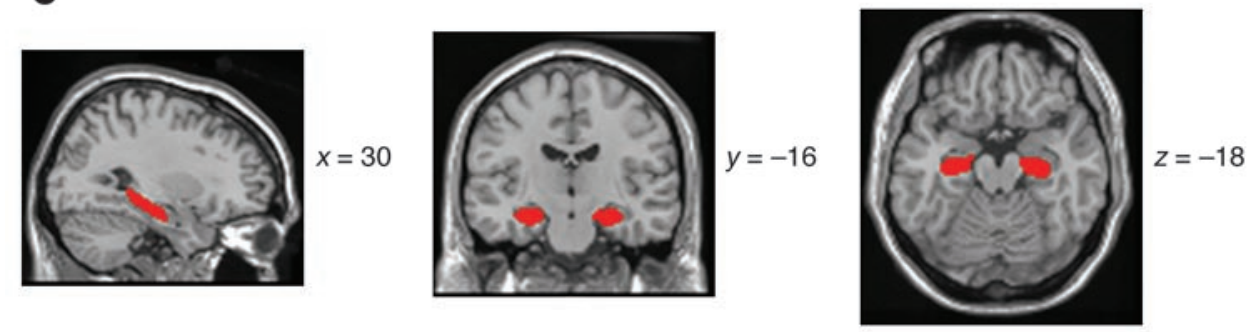

Figure 1

Differences in shape between the HF in WS and normal controls. Shape change is rendered on an average (between groups) template of the HF. (A) Anterior view; (B) posterior view. Color scale correlates to the degree of deformation. Units are arbitrary. Negative values reflect relative local volume reduction in WS patients relative to controls. Positive values reflect local volume expansion in WS patients relative to controls. Double arrow marked A-P shows anterior-posterior direction. Double arrow marked L-R shows left-right direction. Significant findings $(P<0.05$, corrected at the voxel level using small volume correction for the hippocampal ROI) were positive and greater than 0.3 and located in the mid/anterior section of the HF. (C) Same template shown superimposed on a representative single-subject MRI for orientation purposes. Coordinates $x, y$, and $z$ are given in $\mathrm{mm}$ distance from the anterior commissure. 


\section{Table 2}

rCBF, activation, and shape change differences in participants with WS relative to healthy controls

\begin{tabular}{|c|c|c|c|c|}
\hline Area & $\begin{array}{c}\text { Talairach } \\
(x, y, z)\end{array}$ & Z score & $\begin{array}{c}P \text { value } \\
\text { (corrected, } \\
\text { cluster level) }\end{array}$ & $\begin{array}{c}\text { Cluster } \\
\text { size }^{\mathrm{A}}\end{array}$ \\
\hline \multicolumn{5}{|l|}{ PET } \\
\hline Left hippocampus & $-26,-11,-16$ & 5.39 & $<0.001$ & 694 \\
\hline Right hippocampus & $26,-10,-24$ & 4.47 & $<0.001$ & 697 \\
\hline Left occipitoparietal/intraparietal sulcus & $-30,-71,11$ & 3.89 & 0.316 & 78 \\
\hline Right occipitoparietal/intraparietal sulcus & $32,-81,11$ & 3.79 & 0.831 & 14 \\
\hline \multicolumn{5}{|l|}{ fMRI } \\
\hline Left hippocampus & $-30,-11,-19$ & 3.68 & 0.004 & 49 \\
\hline Right hippocampus & $23,-15,-19$ & 3.73 & 0.006 & 42 \\
\hline \multicolumn{5}{|l|}{ Deformation-based morphometry } \\
\hline Left hippocampus & $-32,-28,-18$ & 4.65 & $<0.01$ & 255 \\
\hline \multirow[t]{2}{*}{ Right hippocampus } & $30,-28,-18$ & 2.95 & $<0.05$ & 292 \\
\hline & $20,-4,-32$ & 2.66 & $<0.05$ & 31 \\
\hline
\end{tabular}

Results shown from 3 imaging studies: PET, fMRI, and deformation-based morphometry. Reported significance levels are corrected for either whole-brain volume (PET) or HF volume as defined by the average template created from all study participants (fMRI, morphometry). Coordinates to the left, posterior, and inferior of the commissure are negative. ${ }^{A}$ Cluster size is measured by the number of voxels. the intraparietal/occipitoparietal sulcus, a region that we previously identified in this population (15) as structurally abnormal and relevant for the dorsal stream dysfunction underlying the visuoconstructive deficit in WS (Figure 2B; Table 2).

fMRI. fMRI was used to provide statistical maps showing regions of the brain with significant changes in blood oxygen level-dependent contrast (BOLD). In whole-brain mapping, the BOLD response to passively viewing face and house stimuli compared with viewing scrambled stimuli or baseline showed decreased activation in the parietal part of the dorsal stream, confirming our previous results (data not shown) (20). In the $\mathrm{HF}$, comparison of response to face and house stimuli identified a bilateral lack of activation in the anterior portion in WS, in good regional agreement with the blood-flow reduction observed in PET (Figure 3 and Table diagnostic group, indicating that the physiological asymmetry of hippocampal size was not altered in WS $(\mathrm{F}[1,25]=0.33, P=0.55)$.

Deformation-based morphometry identified significant shape changes located in the midportion of both hippocampi, extending into the dorsal aspect of the hippocampal head (Figure 1 and Table 2). This change corresponded to locally larger structure in WS. Conversely, the data indicated local size reductions in the dorsal HF in WS (Figure 1). However, this difference did not reach statistical significance.

PET. PET imaging provided voxelwise maps of resting relative cerebral blood flow. This identified a highly significant reduction in this parameter in the HF bilaterally, extending into the entorhinal cortex (Figure 2 and Table 2). No other brain region showed abnormalities at the chosen $(P<0.05$, corrected $)$ threshold in the comparison of WS patients with controls. However, at a lower uncorrected threshold of $P<0.001$, we noted hypoperfusion in
2). In this region, the control group activated more for face than house stimuli while participants with WS showed no activation for either stimulus class (Figure 3, insets). ANOVA of responses extracted from the voxel of peak difference in the anterior hippocampus (see Figure 3, insets) confirmed these results by showing a highly significant effect of diagnostic group (reduced BOLD response in WS) (left $\mathrm{F}[1,17]=9.0, P<0.01$; right $\mathrm{F}[1,17]=11.2$, $P<0.005)$ and a significant difference in the differential response to the 2 different stimulus types between diagnostic groups (left $\mathrm{F}[1,17]=4.7, P<0.05$; right $\mathrm{F}[1,17]=6.4, P<0.05)$.

$M R S$. MRS was used to measure the neuronal integrity marker NAA, the ratio of which was normalized to that of total creatine (Cre). A subset of 6 participants with WS were studied using this method and were compared to a set of 6 controls matched for age, IQ, and sex. As described in detail in Methods, voxels with highquality spectra in the HF on the left and right were selected indi-

\section{Figure 2}

Results of PET rCBF mapping. (A) Marked reduction in the anterior $\mathrm{HF}$ bilaterally in participants with WS relative to healthy controls; $P<0.05$, corrected for multiple comparisons. (B) Reduction in rCBF in the intraparietal/occipitoparietal sulcus in WS; $P<0.001$, uncorrected. Coordinates $x, y$, and $z$ are given in $\mathrm{mm}$ distance from the anterior commissure. CTL, control; $T$ score, threshold score.
A

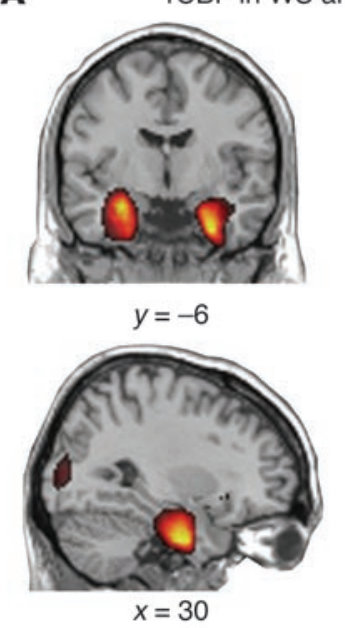

rCBF in WS and CTL participants

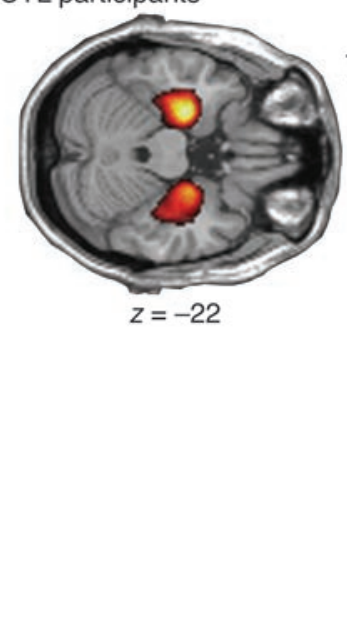

B T score

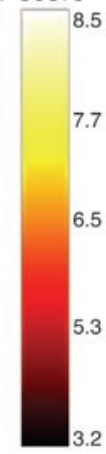


A

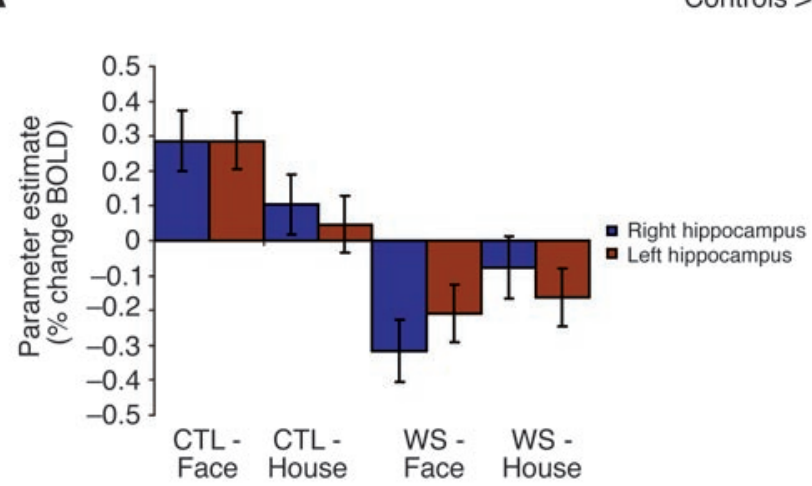

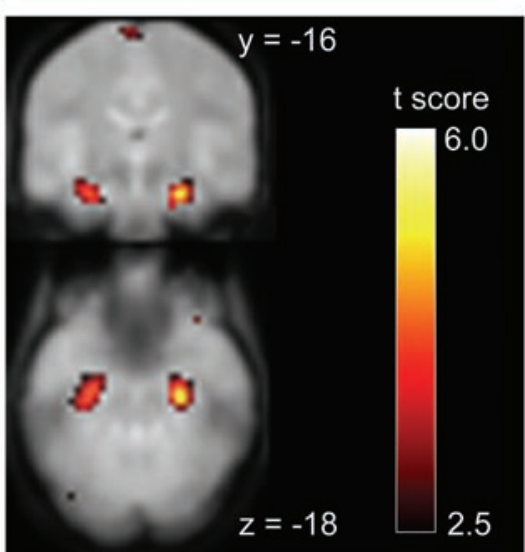

B

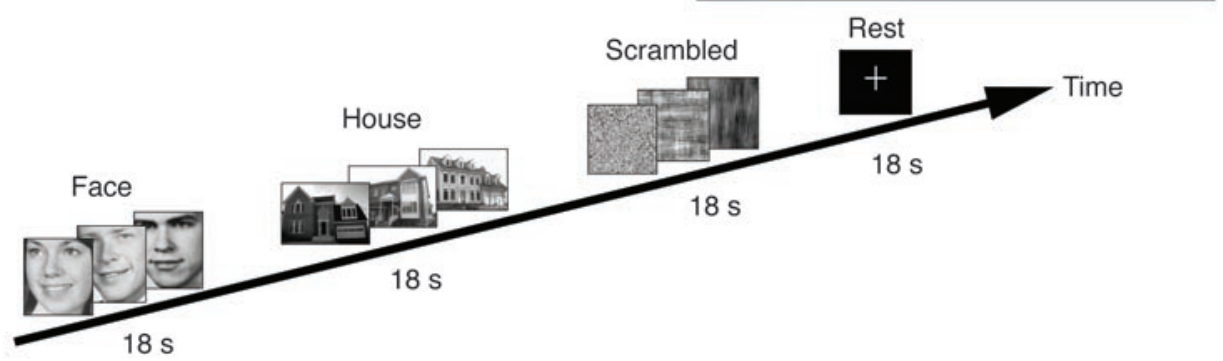

\section{Figure 3}

Results of $\mathrm{fMRI}$ experiment. (A) Average BOLD response to face and house stimuli in participants with WS relative to healthy controls shows reductions in the anterior HF bilaterally, significant at $P<0.05$, corrected for multiple comparisons, shown superimposed on an average fMRI brain volume. Bar graph shows parameter estimates of percentage change in BOLD response to face and house stimuli relative to baseline in both participant groups at the left and right voxels of maximum group difference, located in the hippocampus (see coordinates in Table 3 ). (B) Schema of stimuli and experimental paradigm.

vidually for each subject for this measurement (see Figure 4 for a representative region of interest [ROI], metabolite map, and spectrum). The number of voxels selected (a quality control measure) did not differ between WS and control populations (Table 3). In the left anterior hippocampus, a significant reduction in the NAA/Cre ratio was observed in WS $(17.5 \%$ reduction, $P=0.05$, Mann-Whitney $U$ test; see Table 3 ); the reduction on the right side (11\%) did not reach significance. When data from both sides were combined, we saw a $14 \%$ reduction that also did not reach significance $(P=0.21)$. $\mathrm{NAA} / \mathrm{Cre}$ ratios in a control region in the lateral temporal lobe did not show even a trend toward a group difference (Table 3). Effect sizes (determined by Cohen's $d$ ) were 1.04 in the left hippocampus, a large effect, and 0.45 in the right hippocampus, a medium effect.

\section{Discussion}

Our findings demonstrate striking abnormalities of function and metabolism of the anterior HF in WS. The most pronounced results were obtained while measuring $\mathrm{rCBF}$ during rest, when the anterior hippocampus showed highly significant reductions of flow in WS. Reductions in resting blood flow have been described in disorders having an impact on hippocampal integrity and neuronal function, such as the interictal period of temporal lobe epilepsy (24) or early Alzheimer disease (25). Since excitatory, especially glutamatergic, synaptic activity is prominent in the hippocampus (26) and shown to be altered in the knockout models discussed above $(8,11)$, this finding is in agreement with the preclinical data described and points toward an overall depression of energy metabolism in the HF in WS.
This conclusion is supported by our findings using proton spectroscopy, in which a reduction in the NAA/Cre ratio (more pronounced on the left) was observed in participants with WS relative to controls. NAA/Cre ratios are thought to reflect primarily NAA concentrations, with Cre being assumed constant in most cases (27). NAA is an amino acid found primarily in neurons in the adult CNS and is commonly used as a marker of neuronal viability and energy consumption since synthesis of NAA is localized to neuronal mitochondria, increases linearly with rates of oxygen consumption, and is linked to high-energy phosphate metabolism in the hippocampus (21). Since mitochondrial metabolism is linked to synaptic activity, NAA measures can be viewed as an indirect biochemical proxy for synaptic activity and abundance. In particular, long-term potentiation is highly dependent on intact oxidative metabolism (28), and NAA levels in hippocampus are highly correlated to tissue glutamate concentration (29). Our findings therefore provide convergent evidence for depressed hippocampal energy metabolism and synaptic activity in WS. We noted that the effect size in the left hippocampus was larger than that reported in other diseases where hippocampal function has been assayed using MRS, such as schizophrenia (30), and comparable to that in known hippocampal diseases, such as chronic temporal lobe epilepsy (31). Since the blood flow reduction in this locale was pronounced and bilateral and effect sizes were considerable, we propose that small sample size may be the reason that the reduction on the right side did not reach significance; replication studies using larger cohorts will be informative. 
A

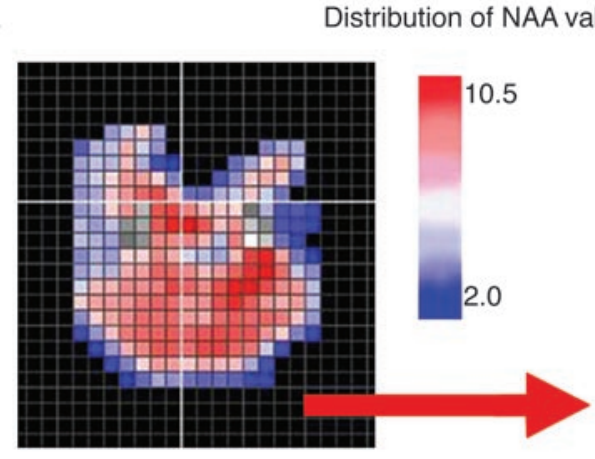

B

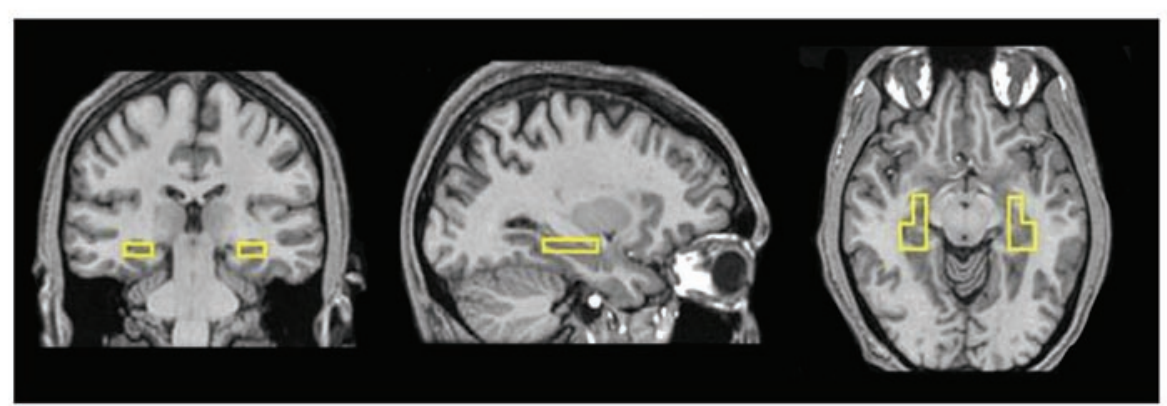

\section{Figure 4}

MR spectroscopy methods. (A) Typical map of the distribution of NAA values (in arbitrary measurement units) at the level of the hippocampus in axial orientation. The visible grid corresponds to the acquisition matrix. A spectrum averaged over all voxels in the ROI with the integration limits for the major metabolites is also shown. (B) Corresponding contour of the employed ROI (6 MRSI voxels on each side) overlaid on this subject's structural MRI showing location at HF. The contour of the $\mathrm{ROI}$ corresponds to the nominal MRSI voxel resolution.
Our finding of a metabolic reduction in the HF at rest in WS has important implications for the functional role of the HF in both WS and normal cognition and is likely linked to the problems in long-term memory, spatial memory, and cognition prominent in this syndrome. Impairment of the anterior hippocampus has been associated with long-term memory deficits in both lesion (32) and human neuroimaging studies (14), and episodic memory function requires interaction with the neocortex, negotiated through the ento/perirhinal cortex (14). Our finding of impaired resting activity in the anterior hippocampus and adjacent entorhinal cortex is therefore in agreement with the deficits in long-term memory observed in WS (16).

Similarly, rodent (12), primate (33), and human neuroimaging studies (34-36) support a role for both the HF and the parahippocampal cortex in spatial navigation (37). However, the prominent abnormalities in visuospatial construction function in WS raise the possibility that HF dysfunction in the spatial domain may be due to deficient processing of visuospatial stimuli in neocortical regions feeding into the hippocampus. For handling visual information, primate cortex is organized into 2 functionally specialized, hierarchically organized processing pathways, a ventral or "what" stream for object processing and a dorsal or "where" stream especially relevant for spatial processing (38). The HF receives prominent projections via parahippocampal cortices from dorsal stream areas, including regions in the parietal lobe (15). According to some models, the parietal lobe may be relevant for maintaining and converting between ego- and allocentric views while the hippocampus proper is important for maintaining an allocentric map of the environment (39). Since we have previously demonstrated a pronounced dysfunction of the dorsal stream in WS underlying the visuospatial construction problems prominent in this disorder (20), a goal of our fMRI paradigm was to disambiguate between a dysfunction in the HF proper and the dorsal stream activation afferent to it.

To this end, we employed face and house stimuli because of their differential processing: while faces preferentially activate the ventral stream, houses are processed by both ventral and dorsal stream regions (40). We reasoned that if hippocampal dysfunction is a consequence of deficient dorsal stream input, HF activation to houses should be selectively impaired whereas abnormal processing within the HF proper should have an impact on both classes of stimuli. We identified a significant activation difference between diagnostic groups in the anterior hippocampus in agreement with the localization of the blood flow reduction found in PET (Figure 3). The response in the anterior hippocampus was clearly impaired for both stimuli in WS

Table 3

MRS results

\begin{tabular}{lcccccccc} 
& $\begin{array}{c}\text { Left } \\
\text { hippocampus } \\
\text { NAA/Cre }\end{array}$ & $\begin{array}{c}\text { No. of } \\
\text { voxels in left } \\
\text { hippocampus }\end{array}$ & $\begin{array}{c}\text { Right } \\
\text { hippocampus } \\
\text { NAA/Cre }\end{array}$ & $\begin{array}{c}\text { No. of } \\
\text { voxels in right } \\
\text { hippocampus }\end{array}$ & $\begin{array}{c}\text { Left } \\
\text { control } \\
\text { region }\end{array}$ & $\begin{array}{c}\text { No. of } \\
\text { voxels in left } \\
\text { control region }\end{array}$ & $\begin{array}{c}\text { Right } \\
\text { control } \\
\text { region }\end{array}$ & $\begin{array}{c}\text { No. of } \\
\text { voxels in right } \\
\text { control region }\end{array}$ \\
WS & $1.60 \pm 0.36$ & $3.33 \pm 1.21$ & $1.72 \pm 0.29$ & $3.43 \pm 0.98$ & $1.96 \pm 0.36$ & $4.29 \pm 1.25$ & $1.92 \pm 0.33$ & $4.43 \pm 1.13$ \\
Controls & $1.94 \pm 0.35$ & $3.50 \pm 0.84$ & $1.93 \pm 0.63$ & $2.71 \pm 1.38$ & $1.83 \pm 0.25$ & $4.57 \pm 0.79$ & $1.89 \pm 0.15$ & $5.29 \pm 2.29$ \\
$P$ value & 0.05 & 0.81 & 0.41 & 0.25 & 0.41 & 0.48 & 0.75 & 0.61 \\
\hline
\end{tabular}

Mann-Whitney $U$ test was used for group comparison. 
Table 4

Demographics and behavioral data

\section{PET and structural data}

\begin{tabular}{|c|c|c|c|c|}
\hline & Sex & Age (yr) & Handedness & IQ \\
\hline Controls & 4 female, 6 male & $30.5 \pm 5.6$ & $100 \%$ right & $99.3 \pm 5.1$ \\
\hline WS & 6 female, 4 male & $29.6 \pm 10.5$ & $100 \%$ right & $93.3 \pm 8.1$ \\
\hline$P$ value & $0.37^{A}$ & $0.81^{\mathrm{B}}$ & & $0.065^{\mathrm{B}}$ \\
\hline \multicolumn{5}{|l|}{ MRSI } \\
\hline & Sex & Age (yr) & Handedness & IQ \\
\hline Controls & 4 female, 2 male & $31.2 \pm 7.8$ & $100 \%$ right & $98.7 \pm 5.5$ \\
\hline WS & 3 female, 3 male & $29.2 \pm 12.0$ & $100 \%$ right & $89.4 \pm 11.7$ \\
\hline$P$ value & $0.56^{\mathrm{A}}$ & $0.71^{\mathrm{B}}$ & & $0.15^{\mathrm{B}}$ \\
\hline \multicolumn{5}{|l|}{ fMRI } \\
\hline & Sex & Age (yr) & Handedness & IQ \\
\hline Controls & 4 female, 6 male & $29 \pm 4.9$ & $100 \%$ right & $97.5 \pm 6.4$ \\
\hline WS & 6 female, 3 male & $31.6 \pm 9.6$ & $100 \%$ right & $92.4 \pm 7.8$ \\
\hline$P$ value & $0.25^{\mathrm{A}}$ & $0.48^{\mathrm{B}}$ & & $0.19^{B}$ \\
\hline
\end{tabular}

${ }^{A} \chi^{2}$ test; ${ }^{B} S t u d e n t ' s t$ test. All participants were right-handed. One control participant was African-American; all others were white, non-Hispanic. Details about IQ measurement are provided in ref. 20.

(Figure 3A), indicating that impaired HF function processing cannot be explained by deficient input from dorsal stream alone.

Instead, our findings suggest a functional deficiency of the HF itself. It will be of interest to explore whether hippocampal dysfunction in WS translates into abnormalities in other brain regions functionally linked to it in the spatial processing stream. One such region is the parahippocampal place area in the medial temporal lobe (41), which receives convergent input from the ventral and dorsal streams and feeds into the HF. In addition, since we did not require a behavioral response during the fMRI scan, our finding might also have been influenced by differences in the attention to the presented stimuli between groups. A recent study of participants with WS and mental retardation and healthy controls using face stimuli indicated hippocampal regional activity and relative increases in posterior hippocampus in WS (42); however, the statistical procedures employed (43) make it unclear whether these results indicate a true difference from the data presented here. Nevertheless, it would be of interest to further explore possible differences in hippocampal function between high-functioning participants with WS and those with mental retardation.

In contrast to the pronounced functional abnormalities, we found that the volume of the HF was preserved in WS. This result agrees with previous data using voxel-based morphometric methods in this population (20) as well as with volumetric results in participants with WS who have mental retardation (44). Preserved hippocampal volume, in agreement with the results from LIMK1 and CYLN2 knockout mice, supports the conclusion that the reduction in blood flow, NAA metabolism, and functional responsivity of the HF is not due to volume loss but rather to functional impairment of neurons in this region. Follow-up neuroimaging studies should be able to clarify the relative impact of the identified abnormalities in the HF on subprocesses of episodic memory and spatial navigation. In addition, neuroimaging studies in participants with WS and mental retardation may identify abnormalities not present in our highly selected study group.
While overall volume was preserved, our analysis of shape using deformation-based morphometry demonstrated an abnormality in the midsection of the hippocampus, extending to the dorsal aspect of the hippocampal head, in WS. As shown in Figure 1, while the midsection and dorsal aspect of the head were relatively larger in WS, the tail was relatively reduced in size, although the latter change did not reach statistical significance. The anterior shape change was adjacent to, but did not fully coincide with, the functional abnormality demonstrated in PET and PMRI. While both anterior and posterior lesions impair spatial navigation in animals, although possibly to different degrees (45), it is noteworthy that humans proficient in spatial navigation (i.e., London taxi drivers) showed increased posterior but decreased anterior hippocampal volume, the opposite pattern from that observed here, suggesting a relationship between the observed shape change and impaired spatial cognition (46).

In summary, our data show impaired metabolism and function of the anterior HF in WS in the setting of preserved volume and subtly altered shape. Our results identify WS as a disorder affecting the HF, provide a neurobiological correlate for the difficulties in long-term memory and spatial navigation prominent in this syndrome, and point toward the involvement in human hippocampal function of genes that are hemideleted in WS. In particular, the agreement of the data presented here with the HF phenotypes of the CYLN2 and LIMK1 knockout mice should prompt further research into the relevance of variation in these genes for normal hippocampal function. Studies in small deletion and atypical deletion individuals $(7,47)$ will be invaluable in further defining the contribution of single genes to hippocampal dysfunction.

\section{Methods}

Participants. Demographics are summarized in Table 4. As outlined previously in a report about a cohort including all WS participants described here (20), participants with WS were chosen after examination of a large sample of adults with WS who had previously participated in behavioral studies conducted by C.B. Mervis. Participants were selected because their IQs were within the normal range for the general population. Exclusionary criteria were chronological age of more than 50 years ( 1 individual excluded) or current treatment with psychotropic medication (1 individual excluded). Of those identified, all but 3 decided to participate in this study. Control subjects were recruited by advertisement and matched groupwise for IQ, sex, and age. Due to time constraints, fewer than half of the participants with WS could be studied using MRS imaging (MRSI). The control groups for the PET and MRI studies differed because many control participants decided to undergo either PET or AMRI, but not both: 5 controls participated in the PET study, but not in any MRI study. Of the controls undergoing MRI, only 6 participated in spectroscopy. All participants gave written informed consent according to the guidelines of the National Institute of Mental Health Institutional Review Board and were reimbursed for their time.

Structural imaging. Acquisition of structural images for this cohort was described previously (20). Six axially acquired T1-weighted structural MRIs (echo time $=5.2 \mathrm{~ms}$, repetition time $=12 \mathrm{~ms}$, field of view $24 \mathrm{~mm}$, resolution $0.94 \times 0.94 \times 1.2 \mathrm{~mm}$ ) were registered and averaged for each participant.

Hippocampi were delineated manually in native space by a trained operator (P. Koch) blind to diagnosis according to the guidelines of Pruessner et al. (48), summarized below. The hippocampal atlas of Duvernoy (49) was also used as a reference. ROI definition was done principally in the coronal plane with reference to the sagittal plane using the software MRIcro (http://people.cas.sc.edu/rorden/mricro.html) (50). 
The posterior border of the HF was defined by the appearance of an ovoid gray-matter mass immediately inferiomedial to the trigone of the lateral ventricle. Two rows of gray matter were excluded laterally unless the trigone and gray-matter border were distinct, in which case all gray matter was included. The medial border in the tail region was defined by drawing a vertical line from the medial border of the lateral ventricle to the parahippocampal gyrus. In the hippocampal body, the lateral border was delineated from the parahippocampal gyrus by excluding the most lateral layer of visible gray pixels. The fimbria and subiculum were included. Superiomedially, the HF bordered the quadrigeminal cistern. The uncal recess and alveus were used as landmarks to aid in the delineation of the lateral, superior, and anterior borders of the hippocampal head. (48). Data were analyzed using ANOVA with diagnostic group as random and laterality as repeated measures factor.

For deformation-based morphometry, an average hippocampal template was created as follows. Each participant's whole-brain gray-matter map was affine normalized to a study-specific gray-matter template previously described (20). This affine mapping was applied to each subject's segmented hippocampi, and the resulting normalized regional maps for all participants were averaged to create a hippocampal template. Each participant's hippocampi were then mapped using high-dimensional nonlinear transformation onto the hippocampal template, and the determinant of the local Jacobian matrix for this transformation was computed for each voxel, resulting in a map of local volume change effected by the transformation to the average hippocampal template for each participant and hippocampus (23). These maps were then compared statistically using a random effects model and thresholded at $P<0.05$, corrected on the voxel level using small volume comparison in the hippocampal average template region. The template was also used for multiple comparison correction of the fMRI data, as described below.

For visualization, an isosurface at the $P=0.5$ probability of overlap level was created in MATLAB (Mathworks) from the average templates and used for display of statistical maps.

MRS. Proton MRSI was performed at 3 tesla (4 slices; spin echo slice selection; repetition time/echo time: $2300 / 280 \mathrm{~ms} ; 7.5 \times 7.5 \times 7.5 \mathrm{~mm}$ voxels) with outer volume suppression to eliminate unwanted lipid signal and no water suppression (51). Following common practice, metabolite signals were calculated as the integral of the magnitude spectrum in a range of $0.25 \mathrm{ppm}$ surrounding peaks for NAA, Cre, and choline (Cho) and were reported as metabolite ratios averaged over the voxels in the ROI (NAA/Cre, NAA/Cho, and Cho/Cre), although this approach means that a contribution of variability in the denominator to the findings is possible. Structural images that were used to prescribe the MRSI sequence and that were in register with the MRSI data were used as a guide to draw the ROIs. Although care was taken to include mostly gray matter in the ROIs, the limited resolution of the technique resulted in inclusion of both gray and white matter in and surrounding the hippocampus (Figure 4). A control region was placed in the lateral temporal lobe bilaterally, a locale where no structural or functional abnormalities have been found in WS by us or others. Only voxels selected to have high spectral quality were used in this analysis, resulting in different numbers of voxels sampled in each subject (see Table 3). Adequacy of spectral quality was assessed by experienced raters (trained to achieve interrater reliability $>0.80$ ), and spectra with line broadening above $9 \mathrm{~Hz}$, poor separation between Cho and Cre, and excessive lipid signal or water signal interfering with the Cho peak were eliminated. Due to the small sample size, the group differences in metabolite ratios were compared using a nonparametric (Mann-Whitney $U$ ) test.

$P E T$. Four resting rCBF scans per participant, each scan following i.v. injection of $12 \mathrm{mCi}$ of oxygen-15 water, were acquired on a GE Advance 3D scanner (septa retracted, $4.25 \mathrm{~mm}$ slice separation, 35 slices, axial field of view $15.3 \mathrm{~cm}$ ), attenuation-corrected, and reconstructed (32 planes,
$6.5 \mathrm{~mm}$ full width at half maximum [FWHM]). Further processing used SPM99 freeware (http://www.fil.ion.ucl.ac.uk/spm). After subtraction of background activity and registration (52), scans were normalized to an average template, scaled proportionally to a whole-brain mean of 50, averaged for each participant, and smoothed $\left(10 \mathrm{~mm}^{3}\right.$ FWHM Gaussian kernel). Average maps were compared between groups using a random-effects model and thresholded at $P<0.05$, whole-brain corrected for multiple comparisons at a height threshold of $T$ equal to 3 .

fMRI. BOLD fMRI was performed on a GE Signa $3 \mathrm{~T}$ using gradient echo planar imaging (EPI) (36 axial slices, $4 \mathrm{~mm}$ thickness, $1 \mathrm{~mm}$ gap, repetition time $/$ echo time $=3000 / 25 \mathrm{msec}$, field of view $=24 \mathrm{~cm}$, matrix $=64 \times 64$ ). Each participant was scanned 3 times for 396 each time, until 128 volume datasets had been collected (first 4 volume datasets excluded). During scanning, participants viewed 18 second blocks of visual stimuli consisting of either houses, faces, scrambled images, or a fixation cross. Sample stimuli are shown in Figure 3. This sequence of 4 conditions was repeated 5 times in each scan. Subjects were instructed to maintain fixation at all times. This was monitored during the scan by an eye-tracking camera that verified that all subjects maintained fixation throughout the scan (Real Eye RE-4501 eye imaging system and SV-7201 Fiber Optic Visual System; Avotec Inc.). For 1 individual with WS, 2 of the 3 scans had to be discarded for excessive movement; exclusion of this participant did not change the significance of the reported group differences in hippocampal activation, and he was retained for the final analysis.

Images were processed as described previously (20) using SPM99. Briefly, images were realigned to the middle image of the scan and spatially normalized into a standard stereotactic space (Montreal Neurological Institute template) using affine mapping followed by nonlinear normalization with $7 \times 8 \times 7$ spatial basis functions. Following standard practice (53), to increase signalto-noise ratio, data were then smoothed with an 8-mm FWHM Gaussian filter and ratio normalized to the whole-brain global mean to remove global variation in AMRI signal. Brain response to each stimulus type (faces, houses, and control stimuli) was modelled as boxcar functions convolved with a synthetic hemodynamic response function and estimated using the general linear model as implemented in SPM99. Statistical images for the contrast of faces or houses versus control stimuli were then obtained for each task and participant and analyzed in a second-level random-effects model (ANOVA and 1-tailed Student's $t$ test) to identify regions where activation differed between diagnostic group for each stimulus class $(P<0.05$, corrected for multiple comparisons within the hippocampal region, as defined by the anatomical template described above). Extracted percentage change in regional BOLD was analyzed using ANOVA and plotted in Figure 3.

\section{Acknowledgments}

We thank the participants with Williams syndrome and their families as well as Neha Dixit, Aaron-Bonner Jackson, Rosanna Olsen, and John Holt for research assistance. This work was supported by the Department of Health and Human Services, the NIH, the National Institute of Mental Health, the Intramural Research Program, and by the National Institute for Neurological Disorders and Stroke grant NS35102 (C.B. Mervis, principal investigator).

Received for publication February 28, 2005, and accepted in revised form April 26, 2005.

Address correspondence to: Andreas Meyer-Lindenberg, 10-4C101, 9000 Rockville Pike, Bethesda, Maryland 20892-1365, USA. Phone: (301) 496-9672; Fax: (301) 496-7437; E-mail: andreasml@nih.gov.

Deepak Sarpal and Paul Koch contributed equally to this work. 
1. Stromme, P., Bjornstad, P.G., and Ramstad, K. 2002. Prevalence estimation of Williams syndrome. J. Child Neurol. 17:269-271.

2. Mervis, C.B., et al. 2000. The Williams syndrome cognitive profile. Brain Cogn. 44:604-628.

3. Klein-Tasman, B.P., and Mervis, C.B. 2003. Distinctive personality characteristics of 8-, 9-, and 10 -year-olds with Williams syndrome. Dev. Neuropsychol. 23:269-290.

4. Chapman, C.A., du Plessis, A., and Pober, B.R. 1996. Neurologic findings in children and adults with Williams syndrome. J. Child Neurol. 11:63-65.

5. Hillier, L.W., et al. 2003. The DNA sequence of human chromosome 7. Nature. 424:157-164.

6. Ewart, A.K., et al. 1993. Hemizygosity at the elastin locus in a developmental disorder, Williams syndrome. Nat. Genet. 5:11-16.

7. Frangiskakis, J.M., et al. 1996. LIM-kinase1 hemizygosity implicated in impaired visuospatial constructive cognition. Cell. 86:59-69.

8. Hoogenraad, C.C., et al. 2002. Targeted mutation of Cyln2 in the Williams syndrome critical region links CLIP-115 haploinsufficiency to neurodevelopmental abnormalities in mice. Nat. Genet. 32:116-127.

9. Hoogenraad, C.C., Akhmanova, A., Galjart, N., and De Zeeuw, C.I. 2004. LIMK1 and CLIP-115: linking cytoskeletal defects to Williams syndrome. Bioessays. 26:141-150.

10. Proschel, C., Blouin, M.J., Gutowski, N.J., Ludwig, R., and Noble, M. 1995. Limk1 is predominantly expressed in neural tissues and phosphorylates serine, threonine and tyrosine residues in vitro. Oncogene. 11:1271-1281.

11. Meng, Y., et al. 2002. Abnormal spine morphology and enhanced LTP in LIMK-1 knockout mice. Neuron. 35:121-133.

12. Rosenzweig, E.S., Redish, A.D., McNaughton, B.L., and Barnes, C.A. 2003. Hippocampal map realignment and spatial learning. Nat. Neurosci. 6:609-615.

13. Zeineh, M.M., Engel, S.A., Thompson, P.M., and Bookheimer, S.Y. 2003. Dynamics of the hippocampus during encoding and retrieval of face-name pairs. Science. 299:577-580.

14. Squire, L.R., Stark, C.E., and Clark, R.E. 2004. The medial temporal lobe. Annu. Rev. Neurosci. 27:279-306

15. Suzuki, W.A., and Amaral, D.G. 1994. Perirhinal and parahippocampal cortices of the macaque monkey: cortical afferents. J. Comp. Neurol. 350:497-533.

16. Vicari, S., Brizzolara, D., Carlesimo, G.A., Pezzini, G., and Volterra, V. 1996. Memory abilities in children with Williams syndrome. Cortex. 32:503-514.

17. Nichols, S., et al. 2004. Mechanisms of verbal memory impairment in four neurodevelopmental disorders. Brain Lang. 88:180-189.

18. Nardini, M., Breckenridge, K.E., Eastwood, R.L., Atkinson, J., and Braddick, O.J. 2004. Distinct developmental trajectories in three systems for spatial encoding between the ages of 3 and 6 years [abstract]. Perception. S33:28b.

19. O'Hearn, K., Landau, B., and Hoffman, J. 2005. Multiple object tracking in people with Williams syndrome and in normally developing children.
Psychol. Sci. In press.

20. Meyer-Lindenberg, A., et al. 2004. Neural basis of genetically determined visuospatial construction deficit in Williams syndrome. Neuron. 43:623-631.

21. Pan, J.W., and Takahashi, K. 2005. Interdependence of N-acetyl aspartate and high-energy phosphates in healthy human brain. Ann. Neurol. 57:92-97.

22. Preston, A.R., Shrager, Y., Dudukovic, N.M., and Gabrieli, J.D. 2004. Hippocampal contribution to the novel use of relational information in declarative memory. Hippocampus. 14:148-152.

23. Gaser, C., Volz, H.P., Kiebel, S., Riehemann, S., and Sauer, H. 1999. Detecting structural changes in whole brain based on nonlinear deformationsapplication to schizophrenia research. Neuroimage. 10:107-113.

24. Tae, W.S., et al. 2005. Cerebral perfusion changes in mesial temporal lobe epilepsy: SPM analysis of ictal and interictal SPECT. Neuroimage. 24:101-110.

25. Kogure, D., et al. 2000. Longitudinal evaluation of early Alzheimer's disease using brain perfusion SPECT. J. Nucl. Med. 41:1155-1162.

26. Bliss, T.V., and Collingridge, G.L. 1993. A synaptic model of memory: long-term potentiation in the hippocampus. Nature. 361:31-39.

27. Jenkins, B.G., et al. 2000. Nonlinear decrease over time in $\mathrm{N}$-acetyl aspartate levels in the absence of neuronal loss and increases in glutamine and glucose in transgenic Huntington's disease mice. J. Neurochem. 74:2108-2119.

28. Izumi, Y., and Zorumski, C.F. 1997. Involvement of nitric oxide in low glucose-mediated inhibition of hippocampal long-term potentiation. Synapse. 25:258-262.

29. Petroff, O.A., Errante, L.D., Rothman, D.L., Kim, J.H., and Spencer, D.D. 2002. Neuronal and glial metabolite content of the epileptogenic human hippocampus. Ann. Neurol. 52:635-642.

30. Bertolino, A., et al. 1996. Regionally specific pattern of neurochemical pathology in schizophrenia as assessed by multislice proton magnetic resonance spectroscopic imaging. Am. J. Psychiatry. 153:1554-1563.

31. Duc, C.O., et al. 1998. Quantitative 1H MRS in the evaluation of mesial temporal lobe epilepsy in vivo. Magn. Reson. Imaging. 16:969-979.

32. Milner, B., and Penfield, W. 1955. The effect of hippocampal lesions on recent memory. Trans. Am. Neurol. Assoc. 1955:42-48.

33. Malkova, L., and Mishkin, M. 2003. One-trial memory for object-place associations after separate lesions of hippocampus and posterior parahippocampal region in the monkey. J. Neurosci. 23:1956-1965.

34. Hartley, T., Maguire, E.A., Spiers, H.J., and Burgess, N. 2003. The well-worn route and the path less traveled: distinct neural bases of route following and wayfinding in humans. Neuron. 37:877-888.

35. Maguire, E.A., Frith, C.D., Burgess, N., Donnett, J.G., and O'Keefe, J. 1998. Knowing where things are: parahippocampal involvement in encoding object locations in virtual large-scale space. J. Cogn. Neurosci. 10:61-76.

36. Shelton, A.L., and Gabrieli, J.D. 2002. Neural cor- relates of encoding space from route and survey perspectives. J. Neurosci. 22:2711-2717.

37. O'Keefe, J., and Dostrovsky, J. 1971. The hippocampus as a spatial map. Preliminary evidence from unit activity in the freely-moving rat. Brain Res. 34:171-175.

38. Ungerleider, L.G., and Mishkin, M. 1982. Two cortical visual systems. In Analysis of visual behavior. D.J. Ingle, D.J. Goodale, and R.J.W. Mansfield, editors. The MIT Press. Cambridge, Massachusetts, USA. 549-586.

39. Burgess, N., Maguire, E.A., and O'Keefe, J. 2002. The human hippocampus and spatial and episodic memory. Neuron. 35:625-641.

40. Grill-Spector, K. 2003. The neural basis of object perception. Curr. Opin. Neurobiol. 13:159-166.

41. Epstein, R., and Kanwisher, N. 1998. A cortical representation of the local visual environment. Nature. 392:598-601.

42. Mobbs, D., et al. 2004. Anomalous brain activation during face and gaze processing in Williams syndrome. Neurology. 62:2070-2076.

43. Poline, J.B., Worsley, K.J., Evans, A.C., and Friston, K.J. 1997. Combining spatial extent and peak intensity to test for activations in functional imaging. Neuroimage. 5:83-96.

44. Reiss, A.L., et al. 2004. An experiment of nature: brain anatomy parallels cognition and behavior in Williams syndrome. J. Neurosci. 24:5009-5015.

45. Broadbent, N.J., Squire, L.R., and Clark, R.E. 2004. Spatial memory, recognition memory, and the hippocampus. Proc. Natl. Acad. Sci. U. S. A. 101:14515-14520.

46. Maguire, E.A., et al. 2000. Navigation-related structural change in the hippocampi of taxi drivers. Proc. Natl. Acad. Sci. U. S. A. 97:4398-4403.

47. Morris, C.A., et al. 2003. GTF2I hemizygosity implicated in mental retardation in Williams syndrome: genotype-phenotype analysis of five families with deletions in the Williams syndrome region. Am.J. Med. Genet. A. 123:45-59.

48. Pruessner, J.C., et al. 2000. Volumetry of hippocampus and amygdala with high-resolution MRI and three-dimensional analysis software: minimizing the discrepancies between laboratories. Cereb. Cortex. 10:433-442.

49. Duvernoy, H.M. 1988. The human hippocampus: an atlas of applied anatomy. J.F. Bergmann Verlag; Springer-Verlag. Munich, Germany. 166 pp.

50. Rorden, C., and Brett, M. 2000. Stereotaxic display of brain lesions. Behav. Neurol. 12:191-200.

51. van Der Veen, J.W., Weinberger, D.R., Tedeschi, G., Frank,J.A., and Duyn,J.H. 2000. Proton MR spectroscopic imaging without water suppression. Radiology. 217:296-300

52. Woods, R.P., Grafton, S.T., Holmes, C.J., Cherry, S.R., and Mazziotta, J.C. 1998. Automated image registration: I. General methods and intrasubject, intramodality validation. J. Comput. Assist. Tomogr. 22:139-152.

53. Frackowiak, R.S.J. 2004. Human brain function. Elsevier Academic Press. Amsterdam, The Netherlands/ Boston, Massachusetts, USA. 1144 pp. 